\title{
Estudio sobre la metodología llevada a cabo en el aula AICLE en un centro educa- tivo público de Tenerife
}

\author{
A Study on the methodology implemented in the CLIL classroom in a public school \\ in Tenerife
}

\author{
Sergio David Francisco Déniz (iD \\ e-mail: sfrancis@ull.edu.es \\ Universidad de La Laguna. España
}

\author{
María Saray Mallorquín Rodríguez \\ e-mail: saraymallorquin@,hotmail.com \\ Universidad de La Laguna. España
}

\begin{abstract}
Resumen
Actualmente existen múltiples formas de abordar metodológicamente el aprendizaje en entornos bilingües, que pueden ser observadas en los centros educativos de todo el país. No obstante, la falta de unificación de criterios sobre la programación de las situaciones de aprendizaje y la implementación de las mismas suele generar unos resultados poco concluyentes sobre su efectividad o solidez y hace necesaria la reivindicación de un plan estratégico estandarizable y extensible a toda la red de centros educativos. Existen diferentes factores que resuenan con mayor fuerza en los resultados y que pueden ser observados en el presente estudio, al igual que en otras investigaciones previas. Así, nos hemos planteado atender cuestiones tales como el grado de motivación y la autoestima/confianza de los aprendices, el enfoque metodológico y la transferencia de aprendizajes a los contextos no formal e informal. El estudio que se presenta se ha desarrollado en un centro educativo público de la isla de Tenerife que lleva a cabo un proyecto AICLE. Para ello, se ha dispuesto de la participación de 121 alumnos y alumnas de la etapa de educación secundaria. En el grupo participante predomina el alumnado de nacionalidad española y una gran mayoría no había formado parte de un grupo CLIL en la etapa educativa o curso anterior. Los resultados obtenidos en esta investigación han mostrado un balance negativo que será comentado posteriormente. Por último, se incluirán unas conclusiones generales a modo de reflexión y cuya intención sirve para replantear una serie de principios que no se están abordando adecuadamente.

Palabras clave: educación secundaria (ESO); metodología AICLE; competencia lingüística; aprendizaje integrado; motivación y contextualización lingüística del aprendizaje.
\end{abstract}

\begin{abstract}
Nowadays, there are multiple ways of applying methodologies in the teaching-learning process in bilingual educational contexts. This fact can be appreciated in schools and high schools all over the country. However, the lack of standardized criteria in planning learning situations as well as in their implementation usually causes inconclusive results about their effectiveness or reliability. Therefore, it is necessary the demand for a strategic plan that can be standardized and applicable to the whole network of schools. There are several factors that stand out in the results which have been observed in previous research as well as in the present study= In this sense, we have set out to pay attention to several issues such as the level of motivation and self-esteem/confidence of the learners, the methodology and the transference of the learning to informal and non-formal contexts. The present research has been carried out in a public school in the island of Tenerife. This public-school counts with a full CLIL project and semi-CLIL project. This group of participants, 121 students of Secondary Education, is characterized by being mostly Spanish and have not been part of a CLIL group in the previous course or group. The results obtained in this research have proved mainly negative and will be analyzed later. Finally, the main conclusions will be laid out to reflect on and suggest a reconsideration of the principles that are not being implemented efficiently and successfully.

Keywords: Secondary Education (ESO); CLIL methodology; linguistic competence; integrated learning; motivation and contextualization of the language of the learning.

Recibido / Received: 14-07-2020

Aceptado / Accepted: 01-04-2021

Publicación en linea / Published online: 28-07-2021
\end{abstract}

Cómo referenciar este artículo / How to reference this article:

Francisco Déniz, S., \& Mallorquín, S. (2021). Estudio sobre la metodología llevada a cabo en el aula AICLE en un centro educativo público de Tenerife. Tendencias Pedagógicas, 38, pp. 148-159. doi: 10.15366.tp2021.38.013. 


\section{Introducción}

Uno de los principales motores que ha impulsado este estudio es la necesidad de identificar las percepciones generales del alumnado sobre buenas prácticas bilingües que tienen lugar en el aula AICLE. Consideramos que si identificamos las percepciones del alumnado AICLE en el aula podríamos disponer de un punto de partida para dar respuesta a esas percepciones adoptando una praxis docente adecuada. De esta forma, sería provechoso prestar atención a los resultados más positivos y satisfactorios estimados por el alumnado, puesto que aportan un feedback concluyente que parte de su propia experiencia educativa.

Aunque el contexto de acción no sea muy amplio ni sea un estudio que se haya replicado para constatar posibles evoluciones favorables o desfavorables en la percepción del alumnado, sí consideramos que puede ser un buen punto de partida para no solo observar esta variable en el tiempo, sino también con otras muestras similares e incluso plantear propuestas metodológicas que incidan en los resultados que aquí se contemplan. Estimamos que la identificación de aquellos aspectos claves considerados/demandados por el alumnado de secundaria para ser tratados en el aula bilingüe ya que, en algunos casos, son obviados dentro de llevar a la práctica una propuesta metodológica u otra: tratamiento del conocimiento, enfoque lingüístico a través del conocimiento técnico, coordinación escolar intercurricular, agrupamientos, nivel de interacción y participación, centros de interés, contextualización del aprendizaje (currículos formal, informal y no formal), evaluación de los aprendizajes, transferencia de los aprendizajes al contexto cotidiano/informal.

A continuación, exponemos los principales objetivos de nuestra investigación:

1. Hallar el grado de satisfacción del alumnado con respecto a su competencia lingüística en lengua extranjera, además de conocer su percepción sobre sus habilidades lingüísticas y curriculares para el seguimiento e interacción en el aula AICLE en su proceso de enseñanza/aprendizaje.

2. Averiguar el pensamiento crítico y reflexivo del alumnado con respecto al grado de participación activa, tipo de distribución (individual, grupos cooperativos, grupos colaborativos, etc.) y dinámicas más habituales en el proyecto AICLE.

3. Descubrir las posibles carencias lingüísticas del alumnado, de acuerdo al nivel de transferencia de los aprendizajes en entornos bilingües a las interacciones y actividades cotidianas/extracurriculares.

Finalmente, este último objetivo debe ser clave para una posible revisión más exhaustiva a nivel metodológico a posteriori.

\section{Marco normativo y planes de acción}

La normativa española que regula de manera específica la mejora y la adquisición de lenguas extranjeras tiene un fuerte impulso en el año 2006 con la Ley Orgánica 2/2006 (LOE, 2006, p. 8). Como se apunta en esa norma, la UNESCO y la Unión europea tenían como objetivo, entre otros, «mejorar el aprendizaje de idiomas extranjeros, aumentar la movilidad y los intercambios y reforzar la cooperación europea».

A nivel estatal y atendiendo a las directrices europeas, la aprobación de la Ley Orgánica de Mejora de la Calidad Educativa, 8/2013 (LOMCE) ha significado un paso adelante en cuanto a políticas de educación plurilingüe. En relación con el fomento del plurilingüismo, la ley indica que este punto es primordial en la educación, ya que resulta ser un pilar fundamental de la sociedad globalizada. Además, la motivación de la adquisición de lenguas extranjeras viene acompañada del uso de recursos tecnológicos como las Tecnologías del Aprendizaje y el Conocimiento (TAC) y en forma de educación competencial que pretende brindar oportunidades al alumnado para desarrollarse tanto en un ámbito local como internacional.

A nivel de la comunidad autónoma de Canarias, la Ley 6/2014, de 25 de julio de Educación no Universitaria establece las bases para lograr un sistema educativo inclusivo y equitativo para el alumnado. El aprendizaje de lenguas extranjeras también es un aspecto que se integra en esta norma 6/2014, se dispone en el artículo 35 el objetivo de formar alumnos y alumnas que sean capaces de comunicarse en los diferentes idiomas que se imparten a lo largo de las etapas educativas, tanto en la primera como en la segunda lengua extranjera. Asimismo, en el artículo 23.7 el Gobierno de Canarias destaca que se fomentarán proyectos para el aprendizaje y adquisición de idiomas y educación CLIL 
(Content and Language Integrated Learning) en la que se integran los contenidos curriculares usando una lengua extranjera como medio vehicular de comunicación. A su vez, el artículo 47 de esta norma 6/2014 propone que los centros que se creen, tanto de educación infantil como de educación primaria, promoverán enseñanzas bilingües, sin embargo, la Educación Secundaria Obligatoria o las enseñanzas de Bachillerato no se incluyen en estos nuevos planes lingüísticos. Aún el artículo número 48 que trata la educación plurilingüe establece que la administración facilitará a los centros la incorporación de materias no lingüísticas usando una segunda lengua, pero llevando a cabo medidas de apoyo para su correcta adquisición. Para lograr estos objetivos que atañen a la educación plurilingüe, es necesario que los docentes estén formados tanto en aspectos lingüísticos como metodológicos (tal y como dispone el artículo 49 de la Ley 6/2014).

Desde el Gobierno de Canarias se han fomentado planes y proyectos que tienen como objetivo mejorar las competencias lingüísticas de la población canaria en la lengua materna y proporcionar competencias plurilingües y multiculturales. En el año 2004 se puso en marcha el Programa Aprendizaje Integrado de Contenidos y Lengua Extranjera (AICLE) que tenía como objetivo integrar la lengua extranjera en la enseñanza de diversas materias en la etapa de educación primaria. Para dar continuidad a este programa se desarrolló el Plan de Impulso de Lenguas Extranjeras de Canarias (PILE). Este plan se instauró en el curso académico 2016/2017 y pretende expandir el objetivo del anterior programa AICLE a todas las etapas educativas (Educación Infantil, Educación Primaria, Educación Secundaria Obligatoria (ESO), Bachillerato, Formación Profesional (FP) y Enseñanzas Oficiales de Idiomas) y atender el aspecto formativo de los docentes. Finalmente, la Consejería de Educación y Universidades, Cultura y Deportes pretende por medio del Plan PILE que el alumnado adquiera de forma gradual al menos una lengua extranjera a través del aprendizaje integrado de contenidos y lengua (AICLE/CLIL). La intención de la administración es ir incorporando progresivamente más centros educativos de las Islas Canarias para lograr educar de forma plurilingüe bajo las directrices del Plan PILE.

Como ha quedado expuesto anteriormente, las medidas adoptadas por las diferentes instituciones gubernamentales con respecto al tratamiento de la educación plurilingüe son relativamente nuevas y el logro de objetivos a nivel macro no será una realidad hasta dentro de unos años aproximadamente. Este hecho sucede de forma similar en la implementación de planes específicos (Proyecto AICLE, Plan PILE) que se han desarrollado durante varios años. En ambos casos, la innovación en política plurilingüe tiene como objetivo último facilitar una mejora o adecuación sustancial de la didáctica al contexto de acción.

El estudio que se ha llevado a cabo, y que detallaremos a continuación, pretende hacer un análisis comparativo de las percepciones y opiniones del alumnado referentes a aspectos metodológicos y lingǘsticos en el aula plurilingüe. Este análisis pretende ser un punto de partida que sirva para posteriormente reflexionar y profundizar sobre qué planteamientos cabría implementar con la intención de propiciar buenas prácticas en la calidad educativa de la enseñanza plurilingüe así como el aprendizaje vivencial del alumnado.

\subsection{Contextualización de la investigación}

Esta investigación se ha llevado a cabo en el IES Andrés Bello en el curso 2019/20. Este centro educativo público se localiza en la zona metropolitana de Santa Cruz de Tenerife, entre los barrios de la Salud, los Gladiolos y el Parque la Granja. El IES Andrés Bello actualmente imparte enseñanzas de Educación Secundaria Obligatoria (ESO) y Bachillerato. Este centro es línea tres y tiene en torno a 600 alumnos/as. El claustro de profesorado está compuesto por 52 docentes, dos profesores comparten centro, siete de ellos comparten turno de mañana y tarde y la mayoría (aproximadamente el $70 \%)$ tienen plaza definitiva en el centro.

La etapa de Educación Secundaria Obligatoria se organiza en tres grupos de 1. ${ }^{\circ} \mathrm{ESO}$, cuatro de $2 .^{\circ}$, cinco de $3 .^{\circ}$ y tres de $4 .^{\circ}$. Entre ellos se encuentran: $1{ }^{\circ}$ del Programa de Mejora del Aprendizaje y del Rendimiento (PMAR) y dos grupos de $2 .^{\circ}$ de PMAR, localizados entre los cursos de $2 .^{\circ}$ y $3 .^{\circ}$ ESO.

Este centro comenzó el proyecto AICLE en el curso escolar 2013/2014 en un grupo de 1. ${ }^{\circ}$ de ESO impartiendo en lengua inglesa las materias: Biología/Sociales, Tecnología e Inglés, debido a que en ese momento disponían de personal acreditado para este tipo de educación. Desde el momento en que comenzaron a desarrollar este proyecto en el centro, cada año se ha incorporado un curso, 
por lo que en el año académico 2014/2015 se integró un grupo de 2. ${ }^{\circ} \mathrm{ESO}$, en 2015/2016 un grupo de $3 .^{\circ}$ ESO y en 2016/2017 un grupo de 4. ${ }^{\circ}$. En el año académico 2017/2018 decidieron incluir en este proyecto AICLE una modalidad denominada semi-AICLE. Esta modalidad consistía en la impartición de una materia usando la lengua extranjera. Esto permitió integrar un grupo más de cada curso y dar así una mayor inclusión al alumnado en este tipo de enseñanzas AICLE.

En la actualidad, en el proyecto AICLE se imparten las siguientes materias: Matemáticas, Tecnología, Biología y Geología, Sociales, Educación Física e Inglés. En cuanto al proyecto semi-AICLE, en $1 .^{\circ}$ ESO se imparte la materia de Tecnología, en $2 .^{\circ}$ ESO Sociales y en $3 .^{\circ}$ y $4 .^{\circ}$ ESO Biología y Geología. No obstante, este proyecto AICLE está en constante proceso de mejora en el centro, puesto que tanto los docentes como el equipo directivo realizan actividades formativas con las que pretenden mejorar su práctica educativa, y también trabajan de forma conjunta en acciones metodológicas y aspectos de innovación educativa.

\section{Planteamiento/Método}

\subsection{Instrumentos}

Para la recogida de datos de esta investigación se ha utilizado como herramienta la encuesta. La encuesta presenta de forma introductoria un espacio denominado «background del alumnado» en el que se busca extraer información relevante del alumnado que participa (Ver Anexo 1). Este apartado permite establecer variables para identificar y contextualizar la muestra que se analiza. Estas variables son: edad, nacionalidad, curso, tiempo que ha estado en el proyecto CLIL, centro de educación primaria de procedencia y si estaba en una clase CLIL anteriormente. Asimismo, a continuación del «background del alumnado» la encuesta se organiza en dos partes claramente diferenciadas. La primera parte, que es en la que se centra esta investigación, consta de doce preguntas cerradas con alternativas, en formato escala de Likert. La escala de Likert es una herramienta que permite medir el grado de conformidad del alumnado participante.

Por tanto, las respuestas alternativas que se presentan a los alumnos y alumnas serían: nada de acuerdo, poco de acuerdo, de acuerdo y muy de acuerdo. Cada variable perteneciente a la escala de Likert se ha traducido en una escala numérica 1-4 (en la que 1 representa la variable «nada de acuerdo» y 4 «muy de acuerdo») para la obtención de datos cuantitativos.

El instrumento que se ha utilizado para el análisis de la confiabilidad de los datos ha sido el modelo del Alfa de Cronbach. Se trata de una medida que permite analizar «la fiabilidad de la consistencia interna de los ítems que forman una escala de medida» (Frias, 2019, p. 3). De este modo, si el valor de alfa se encuentra próximo a 1, la consistencia de los elementos analizados es mayor. Para esta investigación se han considerado finalmente siete elementos que forman parte de la encuesta utilizada en esta investigación. Se ha estimado desechar los elementos 2, 4, 5, 9 y 11 debido a que comprometían la fiabilidad de este estudio. Los elementos seleccionados son: «1. ¿Estás contento con tu nivel de inglés actual?», «3. ¿'Te ves capaz de seguir todas las clases en CLIL?», «6. ¿En las clases CLIL puedes participar más y de forma más activa que en el resto?», «7. ¿Las horas de conversación facilitan y están relacionadas con las clases CLIL?», «8. ¿Es diferente estudiar/trabajar una asignatura en inglés individualmente?», «10. ¿Se realizan proyectos/tareas en las clases CLIL?» y «12. ¿'Te ves capaz de hablar o interactuar con otras personas en inglés fuera del instituto?». (Ver Anexo 1) Después de realizar el análisis, de acuerdo con el alfa de Cronbach la estadística de fiabilidad estima que el valor de los siete elementos analizados es de 0,710 , por lo tanto, es un valor de confiabilidad aceptable.

Tabla 1.

Alfa de Cronbach-Estadísticas de fiabilidad

\begin{tabular}{ll}
\hline Alfa de Cronbach & N de elementos \\
\hline 710 & 7 \\
\hline
\end{tabular}

Fuente: elaboración propia. 


\section{Resultados}

\subsection{Datos y descripción de la muestra}

Esta investigación se ha llevado a cabo en un instituto público de Santa Cruz de Tenerife que imparte enseñanza CLIL/AICLE en la etapa de Educación Secundaria Obligatoria. La muestra de este estudio se basa principalmente en el alumnado que forma parte de los grupos CLIL/AICLE y semi-AICLE del centro. Han participado un total de siete grupos: $1 .^{\circ} \mathrm{B}, 1 .^{\circ} \mathrm{C}, 2 .^{\circ} \mathrm{A}, 2 .^{\circ} \mathrm{B}, 2 .^{\circ} \mathrm{C}, 3 .^{\circ}$ y $4 .^{\circ}$, que computan un total de 121 alumnos y alumnas con edades comprendidas entre los 11 y los 17 años. Tal y como se aprecia en el gráfico de barras que se muestra a continuación, las edades más recurrentes del alumnado participante serían los 13 y 14 años, esto se debe a que en $2 .^{\circ}$ de ESO han participado un total de 45 estudiantes, siendo el curso que mayor muestra obtiene.

Figura 1.

Edad de los participantes

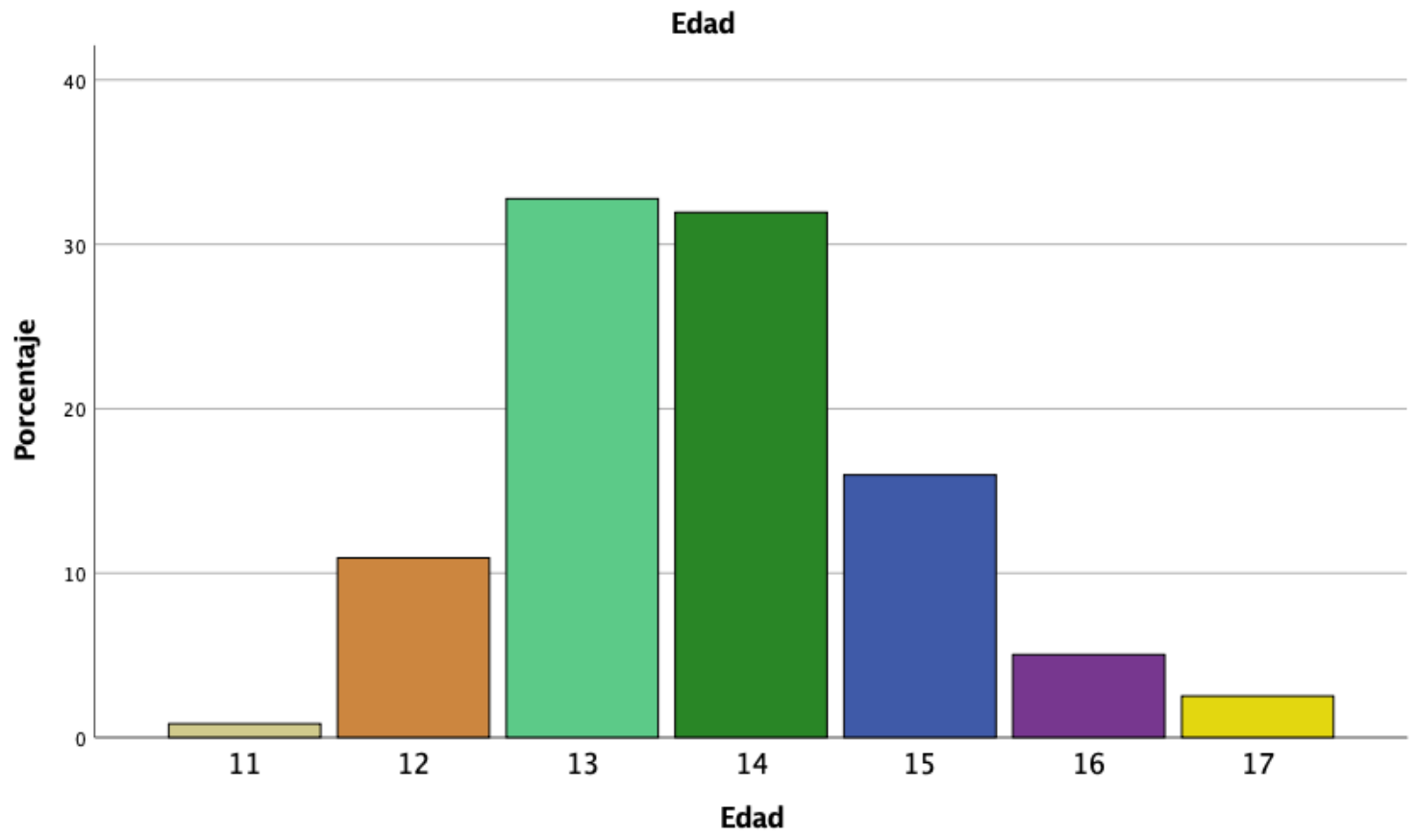

Fuente: elaboración propia.

Debido a la localización urbana del centro educativo donde se desarrolló la investigación, cabe destacar que la muestra de alumnado participante (121 alumnos y alumnas) se organiza en once nacionalidades que aportan riqueza y diversidad cultural (alemán/a, argentino/a, chileno/a, chino/a, colombiano/a, cubano/a, español/a, indio/a, italiano/a, ruso/a y venezolano/a). Es destacable que la mayor muestra referente a la nacionalidad del alumnado pertenece a la nacionalidad española, seguida de la venezolana. Resulta conveniente señalar que para el vaciado de datos de este estudio se optó por no matizar el sexo de los participantes, puesto que no fue una variable que fuésemos a contemplar.

Asimismo, para esta investigación centrada en la educación CLIL era interesante conocer si el alumnado participante había estado en un proyecto educativo CLIL/AICLE en Educación Primaria y, de este modo, analizar los antecedentes educativos del alumnado en lo referente a proyectos educativos bilingües anteriores. Analizando el siguiente gráfico de barras, cabe destacar que el 52,9 \% no estuvieron en una clase CLIL en su etapa de Educación Primaria y, por el contrario, el 31,4 \% respondieron de forma afirmativa. La suma del porcentaje de respuesta afirmativa y el de negativa no alcanza el $100 \%$ de la muestra, sino el $84,3 \%$, esto se debe a que un $15,7 \%$ de la muestra participante no contestó este apartado y se consideran valores perdidos para este ítem. 
Figura 2.

Integración en un programa CLIL

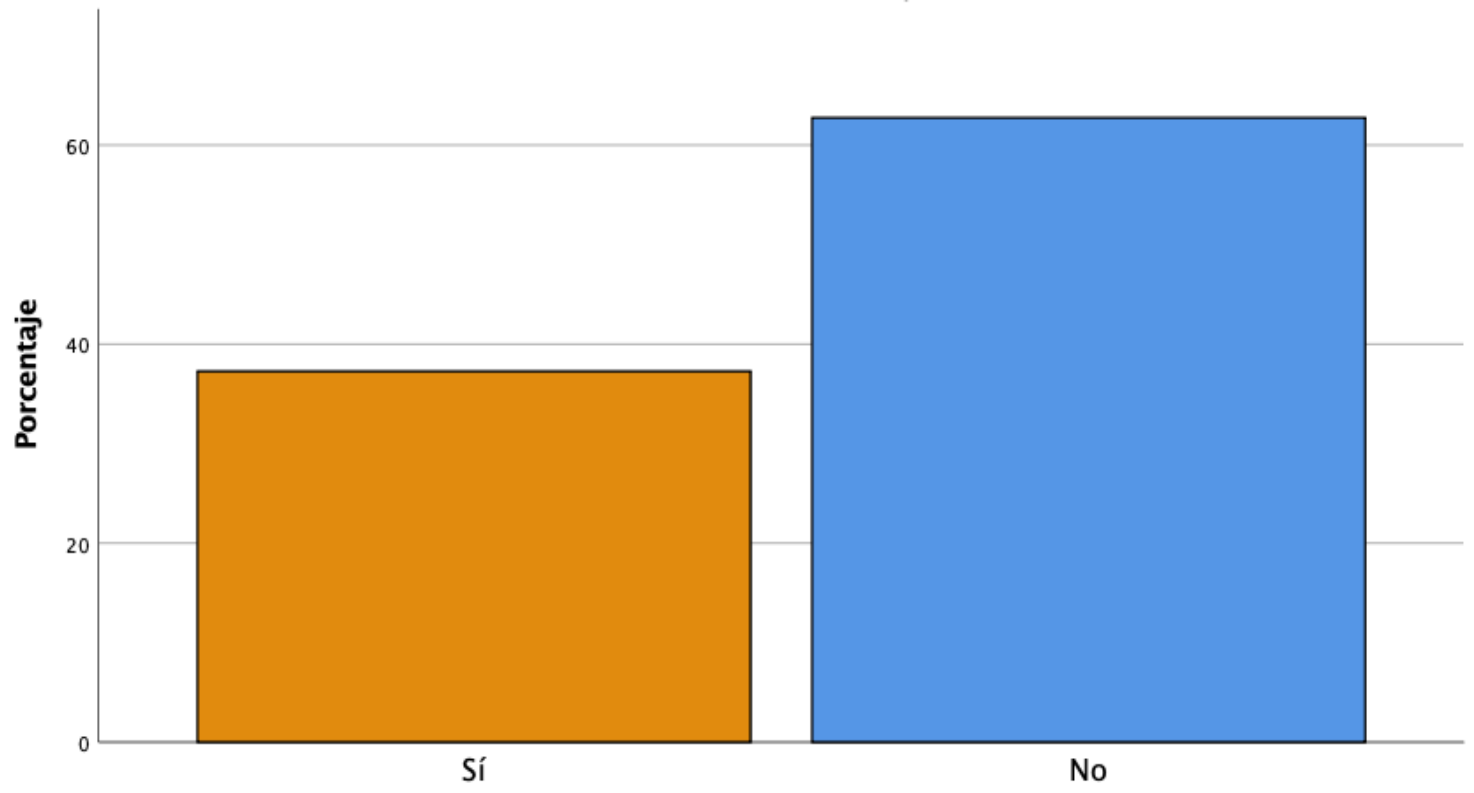

Estabas en una clase CLIL anterior/Primaria

Fuente: elaboración propia.

En conclusión, la muestra participante de este estudio corresponde a un total de 121 alumnos y alumnas del IES Andrés Bello pertenecientes a los grupos AICLE: 2. $.^{\circ} \mathrm{A}, 2 .^{\circ} \mathrm{B}, 3 .^{\circ}$ y $4 .^{\circ}$ y a los semiAICLE: $10^{\circ} \mathrm{B}, 1 .^{\circ} \mathrm{C}$ y $2{ }^{\circ} \mathrm{C}$. Las edades del alumnado participante más frecuentes son los 13 y 14 años y se encuentran en $2 .^{\circ}$ de ESO, siendo el curso con mayor muestra participante. En cuanto a la nacionalidad predominante en el alumnado, es español/a, seguido de venezolano/a. Finalmente, el $52,9 \%$ de los alumnos y alumnas que participaron en esta investigación no habían pertenecido a una clase que recibiera enseñanzas CLIL/AICLE en la etapa de Educación Primaria.

\subsection{Análisis comparativo de la encuesta atendiendo al grupo completo}

A continuación, comenzamos presentando un análisis comparativo de las muestras analizadas, es decir, de los cursos sujetos a las encuestas de satisfacción elaboradas, teniendo en cuenta el grupo completo. En la Tabla 2 se puede apreciar el resultado sobre el nivel de participación ante las preguntas seleccionadas y las medias obtenidas en base a cada una de ellas. Resulta conveniente comentar que el porcentaje arrojado en las medias obedece a la escala de Likert previamente comentada en apartados anteriores, habiendo asignado los valores 1 para «Nada de acuerdo», 2 para «Poco de acuerdo», 3 para «De acuerdo» y 4 para «Muy de acuerdo» (ver Anexo 1 al final).

Tabla 2.

Estadísticos descriptivos del grupo completo

\begin{tabular}{|c|c|c|c|c|c|}
\hline & $\mathrm{N}$ & Mínimo & Máximo & Media & Desviación \\
\hline 1. ¿Estás contento/a con tu nivel de inglés actual? & 121 & 1 & 4 & 2,91 & ,806 \\
\hline 3. ¿Te ves capaz de seguir todas las clases en CLIL? & 121 & 1 & 4 & 2,89 & 964 \\
\hline $\begin{array}{l}\text { 6. ¿En las clases CLIL puedes participar más y de } \\
\text { forma más activa que en el resto? }\end{array}$ & 121 & 1 & 4 & 2,60 & 917 \\
\hline $\begin{array}{l}\text { 7. ¿Las horas de conversación facilitan y están relacio- } \\
\text { nadas con las clases CLIL? }\end{array}$ & 119 & 1 & 4 & 2,71 & ,796 \\
\hline $\begin{array}{l}\text { 8. ¿Es diferente estudiar/trabajar una asignatura en in- } \\
\text { glés individualmente? }\end{array}$ & 119 & 1 & 4 & 2,77 & ,878 \\
\hline
\end{tabular}




\begin{tabular}{|c|c|c|c|c|c|}
\hline 10. ¿Se realizan proyectos/tareas en las clases CLIL? & 120 & 1 & 4 & 3,23 & 896 \\
\hline $\begin{array}{l}\text { 12. ¿Te ves capaz de hablar o interactuar con otras } \\
\text { personas en inglés fuera del instituto? }\end{array}$ & 120 & 1 & 4 & 2,78 & 1,039 \\
\hline $\mathrm{N}$ válido (por lista) & 121 & & & & \\
\hline
\end{tabular}

Fuente: elaboración propia.

A simple vista, es adecuado señalar que los resultados contemplados, teniendo en cuenta todos los cursos, no son del todo positivos y se precisa una reflexión que sirva como punto de partida para la adopción de medidas adecuadas. En primer lugar, cabe destacar que solo hay una pregunta ( $« 10$. ¿Se realizan proyectos/tareas en las clases CLIL?») en la cual la media supera el valor «3. De acuerdo». Esto se puede deber a diferentes causas, aunque sin un conocimiento minucioso de las metodologías aplicadas en las asignaturas bilingües del centro se podría deducir que el empleo de diferentes dinámicas en las que se insta al alumnado a aplicar diferentes conocimientos de forma contextualizada podría motivar la percepción del alumnado con respecto a la respuesta obtenida en este ítem.

Tabla 3.

Media sobre la pregunta 10 del grupo completo

\begin{tabular}{lc|c|c|c|c|c}
\hline & $\mathrm{N}$ & Mínimo & Máximo & Media & Desviación \\
\hline 10. ¿Se realizan proyectos/tareas en las clases CLIL? & 120 & 1 & 4 & 3,23 &, 896 \\
\hline
\end{tabular}

Fuente: elaboración propia.

Sin embargo, tenemos en contraposición la media obtenida para la pregunta «6. ¿En las clases CLIL puedes participar más y de forma más activa que en el resto?». Si realizamos una comparación entre el ítem 10 y el 6 (ver Tabla 4), los resultados son algo contradictorios puesto que la implementación de dinámicas más innovadoras como el uso de proyectos y tareas para el aprendizaje presentan una característica común, que es una mayor implicación por parte del alumnado no solo en la interacción en el aula sino también en el grado de autonomía asumido en el proceso de aprendizaje planteado. Por lo tanto, convendría indagar más sobre esta cuestión confirmando, por una parte, si el alumnado entiende realmente el concepto de proyecto o tarea, y por otra parte, si el profesorado está implementando estas dinámicas o por el contrario usa una terminología que no se corresponde con la praxis.

Tabla 4.

Medias sobre las preguntas 10 y 6 del grupo completo

\begin{tabular}{|c|c|c|c|c|c|}
\hline & $\mathrm{N}$ & Mínimo & Máximo & Media & Desviación \\
\hline 10. ¿Se realizan proyectos/tareas en las clases CLIL? & 120 & 1 & 4 & 3,23 & 896 \\
\hline $\begin{array}{l}\text { 6. ¿En las clases CLIL puedes participar más y de forma } \\
\text { más activa que en el resto? }\end{array}$ & 121 & 1 & 4 & 2,60 & 917 \\
\hline
\end{tabular}

Fuente: elaboración propia

Otra comparación que convendría destacar es la que se puede establecer entre las preguntas «1. ¿Estás contento/a con tu nivel de inglés actual?», «3. ¿Te ves capaz de seguir todas las clases en CLIL?» y «12. ¿Te ves capaz de hablar o interactuar con otras personas en inglés fuera del instituto?». En los tres casos las medias resultantes son inferiores al valor «3. De acuerdo». Aspectos tales como el nivel educativo, la interacción en el aula, el grado de comprensión instruccional, la adquisición de conocimientos y la aplicación de los mismos para la consecución de objetivos de aprendizaje pueden ser factores cruciales para entender la respuesta a la pregunta 3.

Tabla 5.

Medias sobre las preguntas 1, 3 y 12 del grupo completo

\begin{tabular}{ll|l|l|l|l}
\hline N & Mínimo & Máximo & Media & Desviación \\
\hline
\end{tabular}




\begin{tabular}{l|l|l|l|l|l|}
\hline $\begin{array}{l}\text { 1. ¿Estás contento/a con tu nivel de inglés actual? } \\
\text { ¿. ¿Te ves capaz de seguir todas las clases en CLIL? }\end{array}$ 121 & 1 & 4 & 2,91 &, 806 \\
\hline $\begin{array}{l}\text { 12. ¿Te ves capaz de hablar o interactuar con otras per- } 120 \\
\text { sonas en inglés fuera del instituto? }\end{array}$ & 1 & 4 & 2,89 &, 964 \\
\hline
\end{tabular}

Fuente: elaboración propia

En este caso, se puede constatar que la mayoría del alumnado presenta problemas con uno, varios o todos los aspectos anteriormente mencionados. De este modo, la mala percepción de su aprendizaje en un entorno CLIL condiciona indudablemente, en primer lugar, el grado de satisfacción con respecto al nivel de competencia lingüística en lengua extranjera - pregunta 1 - ya que es una forma objetiva y fiable de automedir hasta qué punto el alumnado es competente lingüísticamente (Rodríguez-Pérez, 2012). Esta transferencia podría ser bilateral, ya que si el alumnado considera que posee un nivel de competencia lingüística en lengua extranjera deficiente su enfoque y predisposición en el aula y ante cualquier dinámica en el contexto CLIL podría estar condicionada negativamente. De esta forma se autoimpondría un obstáculo a la hora de poder mejorar y desarrollar nuevas habilidades y estrategias que faciliten su proceso de aprendizaje, mejoren su competencia lingüística y, por ende, no genera una satisfacción personal.

En segundo lugar (ver Tabla 5), pensamos que la media obtenida para la pregunta «12. ¿Te ves capaz de hablar o interactuar con otras personas en inglés fuera del instituto?» está condicionada por las medias de las preguntas 1 y 3 .

Si la percepción propia de la competencia lingüística del alumnado es negativa y su proceso de aprendizaje es accidentado, inefectivo o erróneo, repercutirá en la motivación e iniciativa a la hora de usar la lengua extranjera en entornos comunicativos extraescolares o acceder por voluntad propia a contenidos relacionados con sus centros de interés. Aspectos como la falta de confianza, el miedo al ridículo público, la frustración por la falta de comprensión o la escasez de estrategias propias para una mayor autonomía y flexibilidad tanto dentro como fuera del aula juegan un papel determinante en este sentido. Además, la pregunta 12 condiciona a su vez la respuesta a la pregunta 1 debido a que la percepción de incapacidad comunicativa en entornos reales y en los que, en gran parte, tienden a un uso de la lengua centrado en sus intereses o necesidades puede generar descontento e insatisfacción. De esta forma, se demuestra que estas tres preguntas se retroalimentan entre sí aunque quizás haya que prestar especial atención a la pregunta «3. ¿Te ves capaz de seguir todas las clases en CLIL?» y cómo abordar o replantear la metodología llevada a cabo en el aula para que esas medias no arrojen unos resultados por debajo de lo adecuado en el futuro, y por lo tanto el alumnado se sienta competente lingüísticamente y pueda ampliar su conocimiento teórico y práctico a nivel extracurricular.

Tabla 6.

Media sobre la pregunta 3 del grupo completo

\begin{tabular}{ll|l|l|l|l}
\hline & N & Mínimo & Máximo & Media & Desviación \\
\hline 3. ¿'Te ves capaz de seguir todas las clases en CLIL? & 121 & 1 & 4 & 2,89 &, 964 \\
\hline
\end{tabular}

Fuente: elaboración propia

Si prestamos atención al valor obtenido $(2,71)$ en relación con la pregunta «7. ¿Las horas de conversación facilitan y están relacionadas con las clases CLIL?», podemos dilucidar que de forma generalizada el alumnado considera que las horas de conversación no contribuyen exponencialmente a la mejora de su competencia lingüística en lengua extranjera, a facilitar el trabajo en otra lengua de áreas no lingüísticas, o a ambas.

Tabla 7.

Media sobre la pregunta 7 del grupo completo

\begin{tabular}{l|c|c|c|c|c}
\hline & N & Mínimo & Máximo & Media & Desviación \\
\hline $\begin{array}{l}\text { 7. ¿Las horas de conversación facilitan y están re- } \\
\text { lacionadas con las clases CLIL? }\end{array}$ & 119 & 4 & 2,71 &, 796 \\
\hline
\end{tabular}

Fuente: elaboración propia 
Como hemos expuesto anteriormente, sin tener un conocimiento en profundidad de la gestión e implementación de estas horas orientadas específicamente a la interacción oral, quizás no haya una línea de acción sólida entre las áreas CLIL y las horas de conversación gestionadas por los y las auxiliares de conversación. Convendría enfatizar que, si este trabajo no está conectado directamente con la práctica lingüística llevada en las áreas, más que una ayuda podría suponer un obstáculo e incluso una saturación de contenido para el alumnado. Con esto último no queremos decir que estas horas sean consideradas como clases de apoyo o algo similar, pero sí se precisa de una coordinación fructífera entre ambos agentes educativos, auxiliares y docentes de áreas, para una mejora de los procesos y experiencias de aprendizaje de su alumnado.

Para finalizar con este análisis de resultados nos centraremos en la pregunta «8. ¿Es diferente estudiar/trabajar una asignatura en inglés individualmente?». El hecho de que la media obtenida en esta pregunta refleje que el alumnado, de forma mayoritaria, no está de acuerdo plantea varias cuestiones.

Tabla 8.

Media sobre la pregunta 8 del grupo completo

\begin{tabular}{lll|l|l|l|}
\hline & $\mathrm{N}$ & Mínimo & Máximo & Media & Desviación \\
\hline $\begin{array}{l}\text { 8. ¿Es diferente estudiar/trabajar una asignatura en in- } \\
\text { glés individualmente? }\end{array}$ & 119 & 1 & 4 & 2,77 &, 878 \\
\hline
\end{tabular}

Fuente: elaboración propia

Por un lado, resulta preocupante que el alumnado piense que su aprendizaje en un entorno CLIL no es diferente al que se lleva a cabo en un entorno monolingüe. Si esto es así, resultaría aconsejable hacer una revisión de cómo se están planteando a nivel metodológico las asignaturas CLIL, así como con qué formación cuenta el profesorado en cuestión para poder abordar estos planteamientos didácticos que distan de forma considerable de las asignaturas no lingüísticas impartidas en primera lengua. Si no se está teniendo en cuenta el cambio de paradigma educativo, así como el uso de metodologías más eficaces y que favorezcan tanto la adquisición de la lengua extranjera como del contenido específico del área, es lógico que el alumnado no encuentre diferencias de planteamiento de aprendizajes.

\section{Conclusiones}

Ante la complejidad de la enseñanza plurilingüe que integra contenido curricular y aspectos lingüísticos de la lengua extranjera como medio vehicular comunicativo, es necesario analizar el grado de satisfacción del alumnado con el desarrollo de su habilidad lingüística en lengua inglesa. Este factor puede observarse en los resultados obtenidos en esta investigación, en la que se aprecia que el alumnado no se siente capacitado para seguir las clases CLIL y también se refleja en su grado de satisfacción con respecto al nivel de inglés actual. Las percepciones aportadas por el alumnado nos permiten reflexionar acerca de la comunicación e interacción en el aula. Estas pueden ser tomadas como un punto de partida para estudios posteriores en las que se analicen el tratamiento específico de la competencia lingüística en lengua extranjera, desde su adquisición hasta el desarrollo no solo de destrezas sino también de estrategias, mediante propuestas didácticas concretas y evaluaciones múltiples (en las que se tenga en cuenta no solo la percepción del profesorado sino también la del alumnado desde el punto de vista propio y hacia el compañero, esto es, autoevaluación y coevaluación).

En relación al trabajo en grupos y a la realización de proyectos y tareas con objetivos comunes de aprendizaje, los resultados obtenidos en el estudio indican que el alumnado entrevistado no diferencia claramente entre un aprendizaje individualizado y un aprendizaje por grupos. Opinamos que los y las aprendices deben ser capaces de distinguir entre ambos tipos de agrupamientos, que suelen ir ligados a prácticas más específicas en función de uno u otro. Sin embargo, de forma generalizada se observa que los proyectos y las tareas son dinámicas habituales en el aula CLIL, lo cual no garantiza que se realicen de forma eficiente o atendiendo a un enfoque de aprendizaje integrado propio de un entorno bilingüe. Por ello, destacamos que de acuerdo a los resultados obtenidos el alumnado está realizando proyectos y tareas, no obstante, desconocemos si se trabaja por proyectos y tareas. Estas metodologías implican un enfoque protagonista del alumnado con una participación activa y autónoma en el aula, sin embargo, el alumnado aportaba una respuesta por debajo del «adecuado» a esta cuestión. 
Para una valoración más precisa y sustancial acerca de esta cuestión, se estima oportuno indagar más sobre los proyectos y tareas que se realizan en el centro grupo por grupo y así extraer unas reflexiones que puedan contribuir a una posible revisión metodológica futura.

Otra reflexión que estimamos oportuna sería la que concierne a la insuficiente transferencia de aprendizajes llevados a cabo en el aula y percibidos por el alumnado participante en nuestro estudio. Consideramos que en este punto en concreto es el alumnado en sí el que mejor feedback puede dar al respecto, ya que son ellos y ellas los que luego, en su horario extraescolar, pueden poner en práctica sus aprendizajes, ampliarlos y reflexionar acerca de los mismos en diversas situaciones de ámbito extracurricular. Este indicador puede servir al profesorado para reflexionar sobre ciertas dinámicas en la educación plurilingüe. Tal y como afirma Rodríguez-Pérez (2012, p. 384): «El estudio de un idioma puede hacer emerger vivencias negativas o positivas ocasionando respuestas condicionadas». Resulta conveniente resaltar la apreciación de una valoración negativa en las interacciones en segunda lengua podrían incidir en la autoestima y seguridad de los aprendices, además de afectar a la motivación intrínseca y generar como resultado un rechazo a continuar su aprendizaje centrado en sus propios intereses personales y sociales fuera del ámbito escolar.

A modo de conclusión, en este estudio se ha podido establecer una serie de valoraciones y reflexiones teniendo en cuenta un contexto muy específico, pero que no por ello puede ser extrapolable a la realidad imperante en muchos de los centros de la geografía española. El paso siguiente para profundizar en la cuestión que aquí nos concierne sería plantear un sondeo similar en otros centros de características o realidades distintas. De esta forma, se podrían tomar en consideración las percepciones más comunes sobre los diferentes aspectos que hemos señalado como cruciales para, a continuación, cuestionar y revisar metodologías mediante la implementación y evaluación de dinámicas concretas en el aula AICLE.

\section{Referencias}

Frias-Navarro, D. (2019). Apuntes de consistencia interna de las puntuaciones de un instrumento de medida. Universidad de Valencia. España. Recuperado de https://www.uv.es/friasnav/AlfaCronbach.pdf

Ley Orgánica 2/2006, de 3 de mayo, de Educación. Boletín oficial del Estado, 106, de 4 de mayo de 2006, pp. 17158 a 17207. Recuperado de https://www.boe.es/eli/es/lo/2006/05/03/2

Ley Orgánica 8/2013, de 9 de diciembre, para la mejora de la calidad educativa. Boletín oficial del Estado, 295, de 10 de diciembre de 2013, pp. 97858 a 97921. Recuperado de https://www.boe.es/eli/es/lo/2013/12/09/8

Ley 6/2014, de 25 de julio, Canaria de Educación no Universitaria. Boletín Oficial de Canarias, 152, pp. 21133 a 21200. Recuperado de http://www.gobiernodecanarias.org/boc/2014/152/002.html

Resolución de 2 de julio de 2019 de la Dirección General de Ordenación, Innovación y Promoción Educativa, por la que se dictan instrucciones a los centros públicos de educación infantil y primaria de la comunidad autónoma de Canarias autorizados a impartir la enseñanza bilingüe dentro del marco del plan de impulso de lengua extranjeras en el curso 2019-2020. Recuperado de https://www.gobiernodecanarias.org/cmsweb/export/sites/educacion/web/galerias/descargas/normativa-internas/resolucion instrucciones bilingue 19 20.pdf

Resolución de 26 de agosto de 2019 de la Dirección General de Ordenación, Innovación y Calidad, por la que se dictan instrucciones para el desarrollo de la modalidad de aprendizaje integrado de contenidos y lenguas extranjeras (Programa AICLE) en centros educativos públicos que imparten enseñanza de régimen general en la comunidad autónoma de Canarias para el curso 2019-2020 y se publica el listado de los centros educativos públicos autorizados. Recuperado de https://www.gobiernodecanarias.org/cmsweb/export/sites/educacion/web/_galerias/descargas/normativa-internas/instruciones_aicle_19_20.pdf

Rodríguez-Pérez, N. (2012). Causas que intervienen en la motivación del alumno en la enseñanzaaprendizaje de idiomas: el pensamiento del profesor. Didáctica Lengua y Literatura, 24, pp. 381-409. Recuperado de https://revistas.ucm.es/index.php/DIDA/article/view/39932. doi: 10.5209/rev_DIDA.2012.v24.39932 
Anexo A. Encuesta para el alumnado CLIL/AICLE, páginas 1-2

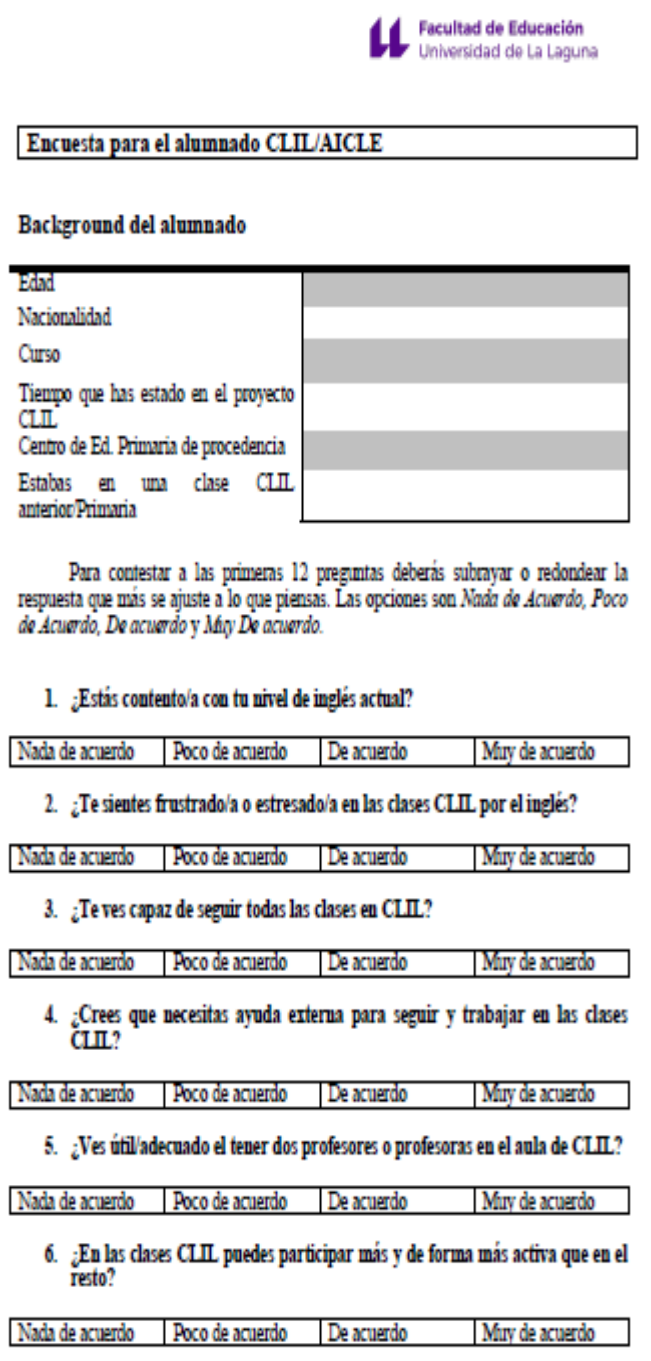

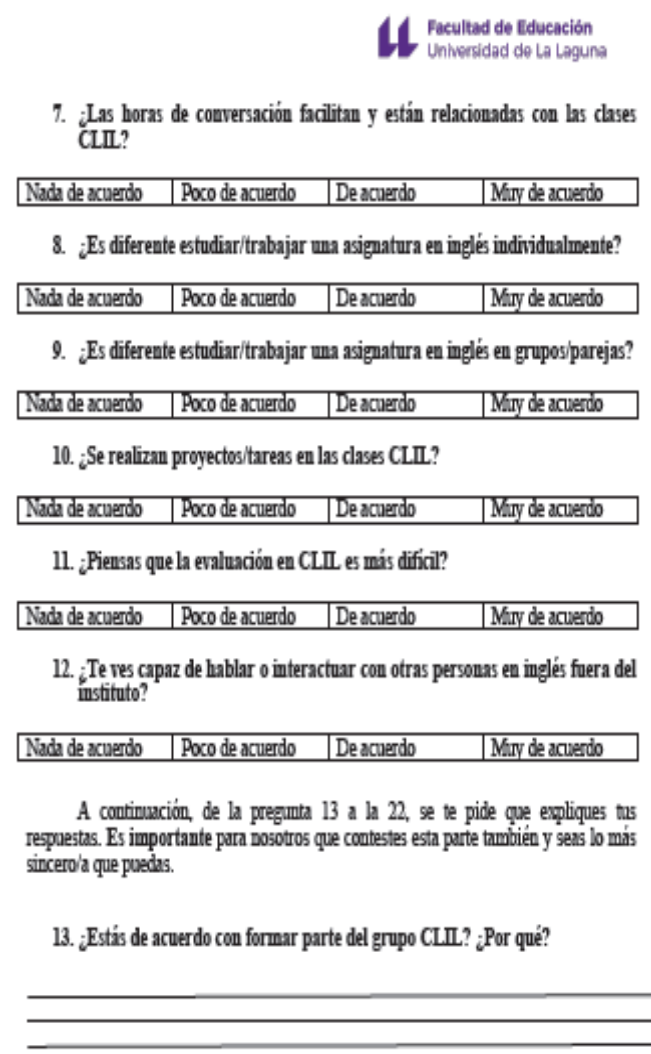

14. En las clases CLII, te relaciouas mejor 0 peor con tas compañerosicompañeras? Desarrolla tu respuesta.

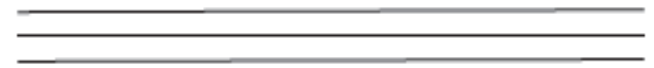

15. ¿Qué asignaturas son más dificiles de seguir en inglés? Dinos cuáles. 
Anexo B. Encuesta para el alumnado CLIL/AICLE, página 3

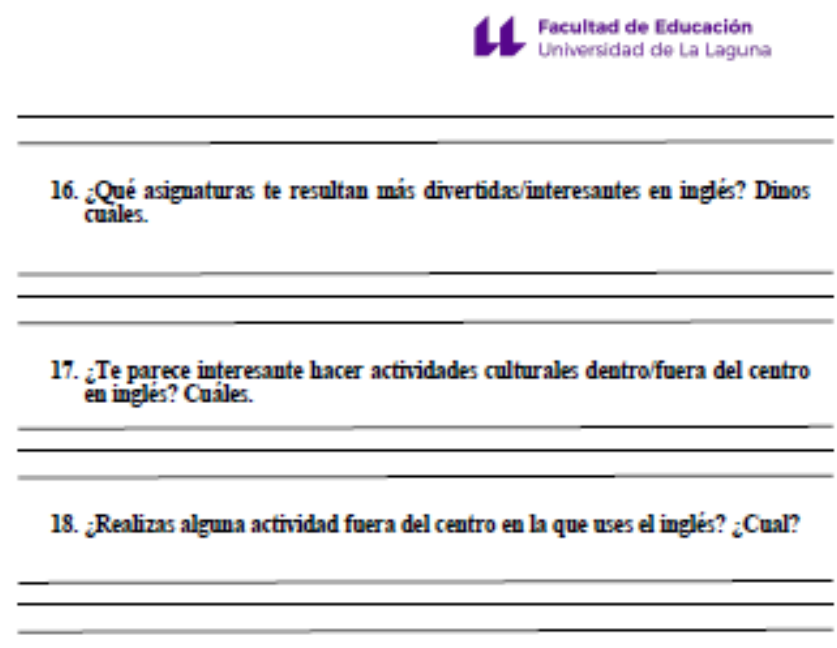

19. ¿Qué cosas cuentan para tu calificación en CLII? (cuaderno, actividades. eramenes, proyectos, autoevaluación, evaluar al companiero, etc.)

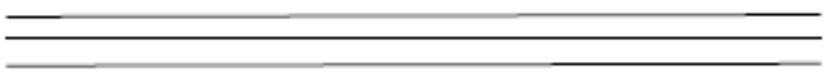

20. Estar en un grupo CIIL te ha animado a usar las redes sociales/ver peliculas/jugar a juegos/leer en inglés en tu tiempo libre? Desarrolla to respuesta.

21. ¿Qué destacarias de las clases CLIIL?

22. ¿Qué cosas ves mejorables en las clases CIII? 